\title{
Measurements of ultra wide band in-vehicle channel - statistical description and TOA positioning feasibility study
}

\author{
Jiri Blumenstein ${ }^{1 *}$, Ales Prokes ${ }^{1}$, Tomas Mikulasek ${ }^{1}$, Roman Marsalek ${ }^{1}$, Thomas Zemen ${ }^{2}$ \\ and Christoph Mecklenbräuker ${ }^{1,3}$
}

\begin{abstract}
This paper reports on a real-world wireless channel measurement campaign for in-vehicle scenarios in the UWB frequency range of 3 to $11 \mathrm{GHz}$. The effects of antenna placement in the vehicle's passenger compartment as well as the effects due to the presence of passengers are studied. The measurements have been carried out in the frequency domain, and the corresponding channel impulse responses (CIRs) have been estimated by inverse Fourier transform. The influence of a specific band group selection within the whole UWB range is also given. Statistical analysis of the measured channel transfer functions gives a description of the wireless channel statistics in the form of a generalized extreme value process. The corresponding parameter sets are estimated and documented for all permutations of antenna placement and occupancy patterns inside the vehicle's passenger compartment. Further, we have carried out a feasibility study of an in-vehicle UWB-based localization system based on the TOA. The positioning performance is evaluated in terms of average error and standard deviation.
\end{abstract}

Keywords: UWB; In-vehicle environment; Channel model; Positioning; TOA

\section{Introduction}

The onboard electrical power distribution, communication, and networking functionalities are realized by cable bundles in today's vehicles. We observe a trend towards increasing numbers of sensors, actuators, control units, and infotainment systems in cars and trucks. As a direct result, the weight of the wiring in all types of vehicles increases. Moreover, their flexible installation and reliability represent a challenging and costly task [1]. The weight of the wiring becomes even more serious when the vehicles are powered fully electrically.

In $[2,3]$, the authors conclude that ultra wide bandwith (UWB) technology with its favorable radio environment characteristics for indoor areas such as low transmit power and robustness against multipath fading could be extrapolated even for the vehicular passenger compartment. Naturally, attempts to replace cable bundle start

\footnotetext{
*Correspondence: blumenstein@feec.vutbr.cz

1 Department of Radio Electronics, Brno University of Technology, Technicka 12,61200, Brno, Czech Republic

Full list of author information is available at the end of the article
}

up with in-vehicle radio channel measurements were performed by authors in [4-9] and by channel modeling in $[3,10]$, and a clustering approach for intra-bus channel modeling is studied in [11,12]. Attempts to build a prototype of an UWB-based wireless sensor network within a vehicle, both in the passenger and the engine compartments, are published in $[13,14]$. In [15], the topic of wireless in-vehicle communication links based on LTE is discussed while reckoning with specific in-vehicle impulse noise. In [16], the UWB channel inside a vehicle is studied from a spatial stationarity point of view. The necessity of detailed knowledge of the channel characteristics is of highest importance for the proper physical layer design of any wireless communication system.

Together with this motivation to substitute at least part of the vehicle's cable bundles by wireless links, a wireless localization service within the vehicle is desirable. Future applications of such a localization service include remote keyless entry and ignition systems, advanced child passenger safety, and beamsteering for in-vehicle high-speed Internet access.

\section{Springer}


In [14,17], a localization service utilizing UWB is studied and it is concluded that in general, thanks to the high time resolution of UWB impulses, the time of arrival (TOA) technique is capable of providing sufficient spatial resolution for a variety of applications. Although the TOA works reliably in environments with LOS, it can be used with some restriction also in NLOS scenarios. In the multipath environment, the important component for ranging based on the TOA technique is the direct ray, which propagates from the transmitter towards the receiver. When the beam penetrates some obstacles whose attenuation does not avoid the beam detection, the TOA technique is applicable. Note that, for example, in the US, the frequency range of 1.99 to $10.6 \mathrm{GHz}$ is deregulated for communications and wall-penetrating radars, enabling looking into or through non-metallic materials $[14,18]$. Thus, the presumption is that even the harsh in-vehicle ambiance with OLOS propagation may provide sufficient positioning accuracy.

In $[4,6,7,19]$, the path-loss, seat material, and occupancy influences are presented for the frequency range of 3 to $8 \mathrm{GHz}$. Since the positioning service deployment is not seen as the aim of $[4,6,7,19]$, the placement of transmit antennas is inappropriate from that point of view. Thus, resulting parameters could differ from parameters obtained by measurement campaigns which take the positioning into account in the first place.

\subsection{Contribution of the paper}

While taking into account the influence of the occupancy, antenna placement, and the influence of a specific frequency band group selection, in this paper, we address the following:

- Intra-vehicle channel measurement and statistical evaluation via GEV. This allows a reproducibility of the measured results for 90 selected wireless links within a passenger car compartment.

- Statistical analysis and the in-vehicle positioning in the UWB range of 3 to $11 \mathrm{GHz}$. The aim of the article is to give a general overview of the achievable accuracy of ranging regardless of LOS and NLOS scenarios.

The paper is organized as follows. In Section 2, we provide an overview of our measurement site including a hardware description. In Section 3, we present our channel measurement tools including our conical monopole antenna design [20] and we define the sought channel parameters. In Section 4, the feasibility of the positioning service deployment within a vehicle compartment is assessed, while the conclusion in Section 5 sums up the paper.

\section{Measurement setup}

\subsection{Measurement bandwidth and dynamic range}

The scheme of the measurement setup is shown in Figure 1. The complex CIRs (below introduced by (1)) corresponds to the $s_{41}, s_{42}$, and $s_{43}$ scattering parameters which are measured in the frequency domain for two different frequency bands, 3 to $11 \mathrm{GHz}$ (entire UWB band with a bandwidth of $B=8 \mathrm{GHz}$ ) and 3.3168 to $4.752 \mathrm{GHz}$ (first band group with a bandwidth of $B=1.58 \mathrm{GHz}$ ), utilizing a four-port vector network analyzer Agilent Technologies E5071C (VNA; Agilent Technologies Inc., Santa Clara, CA, USA) (Figure 2). The spatial placement of the receiving (RX) and transmitting (TX) antennas inside the vehicle is depicted in Figure 3.

The dynamic range of the measurement setup is higher than $90 \mathrm{~dB}\left(P_{\text {outVNA }}=5 \mathrm{dBm}\right.$, IF bandwidth $\left.=100 \mathrm{~Hz}\right)$. The chosen frequency step of $10 \mathrm{MHz}$ results in 801 frequency points in the case of the entire UWB band and 159 frequency points in the case of the first band group.

In order to avoid a degradation of the measured phase accuracy due to movements of the RX antenna, phasestable coaxial cables were used and included in the calibration process. The measurement is carried out in the Skoda Octavia 1.8 TSI car.

\subsection{Antenna placement}

As depicted in Figure 3, the RX antenna is placed at various locations inside the car compartment (on all seats and in the boot) and the TX antennas are placed on the left and right sides of the dashboard, top corners of the windshield, and at the rear part of the ceiling.

The channel measurements are carried out for both LOS and NLOS scenarios. NLOS is caused by the backrest of the seats, the dashboard, and/or persons sitting inside the vehicle.

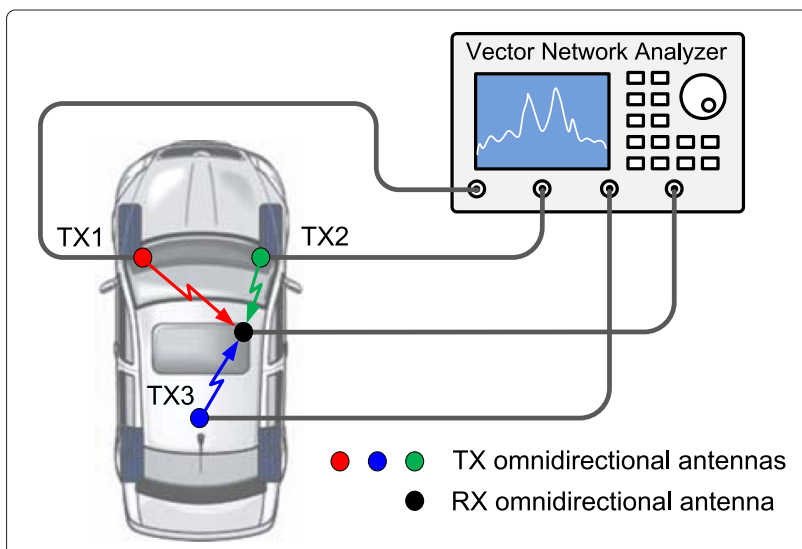

Figure 1 Measurement setup containing the vector network analyzer Agilent Technologies E5071C and the car Skoda Octavia 1.8 TSI. 


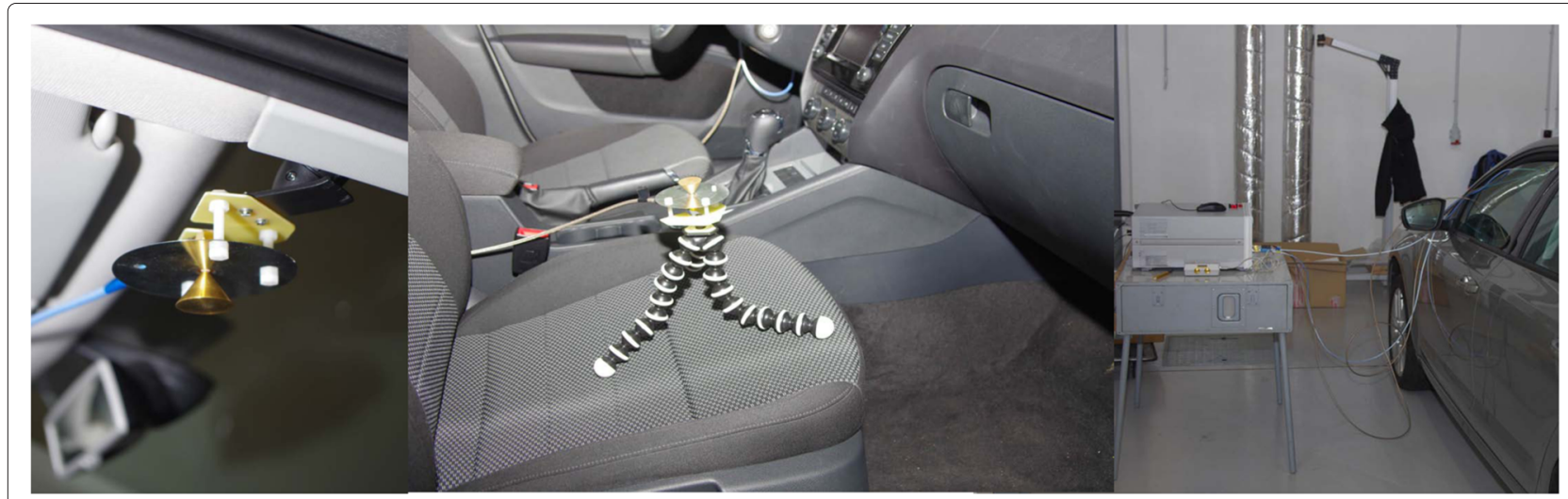

Figure 2 Images of the conical monopole antenna, measurement position, and four-port VNA. [left] Detail of the conical monopole antenna mounted on the front windshield. [middle] One measurement position inside the vehicle. [right] Four-port VNA connected with antennas inside the measured vehicle.

Since the radiation pattern of the conical monopole antenna [20] is very close to the omnidirectional radiation pattern, we were able to capture a maximal number of multipath components (reflected waves).

In Figure 4, the conical monopole has an omnidirectional $\mathrm{H}$-plane radiation pattern which is invariant in the frequency band of interest. Due to a variable gain in the lower half E-plane radiation pattern (elevation angle from $90^{\circ}$ to $-90^{\circ}$ ), the antennas were placed in the car compartment so that the upper half E-plane radiation pattern (almost constant) was used. It means that when the antenna was placed at the cabin ceiling, it was situated bottom up. Thus, the LOS and NLOS are minimally affected by the radiation pattern; however, with the reflected waves arriving from the TX antenna or incident on the RX antenna, the lower elevation angle might be affected by the non-ideal radiation pattern of the antennas.

\section{Channel parameters}

The CIR describes the wireless channel. We utilize an inverse discrete Fourier transform of the windowed scattering parameter series, expressed as:

$$
h^{\alpha}(n)=\sum_{k=0}^{N-1} w(k) s_{\zeta}^{\alpha}(k) e^{j k n 2 \pi / N},
$$

where $s_{\zeta}^{\alpha}(k)$ corresponds to the $k$ th measured scattering parameter (as described in Section 2) and $w(k)$ represents the Blackman window. Parameter $\alpha$ denotes the spatial positions of the transmit and the receive antenna in the measured vehicle and $\zeta \in\{41,42,43\}$. For practicality in the following statistical processing, we arbitrarily merge indices $\alpha$ and $\zeta$ into one measurement number $\alpha \in\{1, \ldots, 90\}$. Hence, in the following, it is not possible to assign the specific measured data to the actual spatial positions.

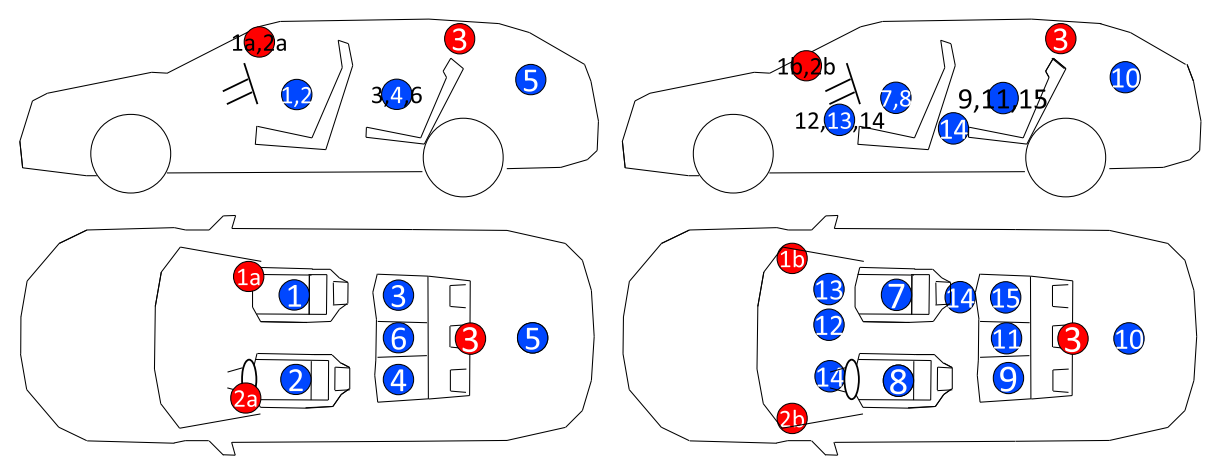

Figure 3 The positions of transmitting (red) and receiving (blue) antennas. We employ two possible receive antenna placement patterns. As seen on the left part, the antennas $1 \mathrm{a}$ and $2 \mathrm{a}$ occupy the left and right top corners of the windshield, while on the right part, the antennas $1 \mathrm{~b}$ and $2 \mathrm{~b}$ are positioned in the lower corners. Please note that all measurements have been measured for various passenger layouts. We have considered (1) empty vehicle and (2) driver and two to three passengers. 


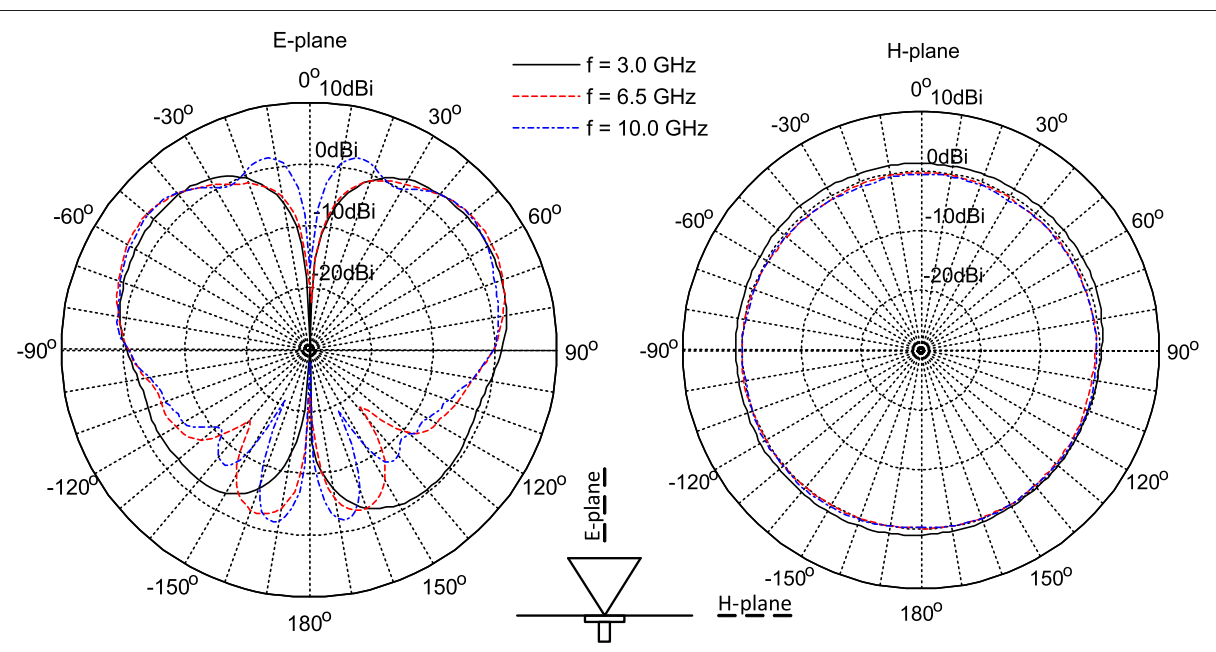

Figure 4 Measured gain pattern of the conical monopole antenna [20].

The number of measured frequency points $N=801$ for the entire UWB or $N=159$ for the first band group. Since the in-vehicle channel is assumed to be time invariant, we performed one repetition of the scattering parameter measurement.

The relationship between discrete time delay $n$ and continuous time delay $\tau$ is given by:

$$
\tau_{n}=n \frac{1}{B}
$$

where $1 / B$ stands for the time resolution (see Equation 7 ).

For a statistical characterization of the UWB channel, we use the MDP which is defined as the magnitude of complex CIR:

$$
A(\tau)=|h(\tau)| .
$$

\subsection{Statistical description of the received signal}

\subsubsection{Independent identically distributed (IID) phase}

In this chapter, the phase statistics of the measured CIRs are presented. As visible in Figure 5 [right], according to the ecdf evaluated for each measured CIR, the phase $\Phi^{\alpha}(\tau)$ is uniformly distributed.

Moreover, utilizing the Pearson correlation coefficient $\rho_{\alpha, \beta}$, we evaluate the mutual dependence between phases for all measured positions denoted as $\alpha$ and $\beta$. The Pearson correlation coefficient is given as:

$$
\rho_{\alpha, \beta}=\frac{\mathrm{E}\left[\left(\Phi^{\alpha}(\tau)-\xi^{\alpha}\right)\left(\Phi^{\beta}(\tau)-\xi^{\beta}\right)\right]}{\wp^{\alpha} \wp^{\beta}}
$$

where $\wp^{\alpha}$ denotes the standard deviation and $\xi^{\alpha}$ the mean of $\Phi^{\alpha}(\tau)$. The evaluation of the Pearson correlation coefficient is visible in Figure 5 [left] showing uncorrelated behavior of $\Phi^{\alpha}(\tau)$. The operator $\mathrm{E}[\cdot]$ denotes the expected value.
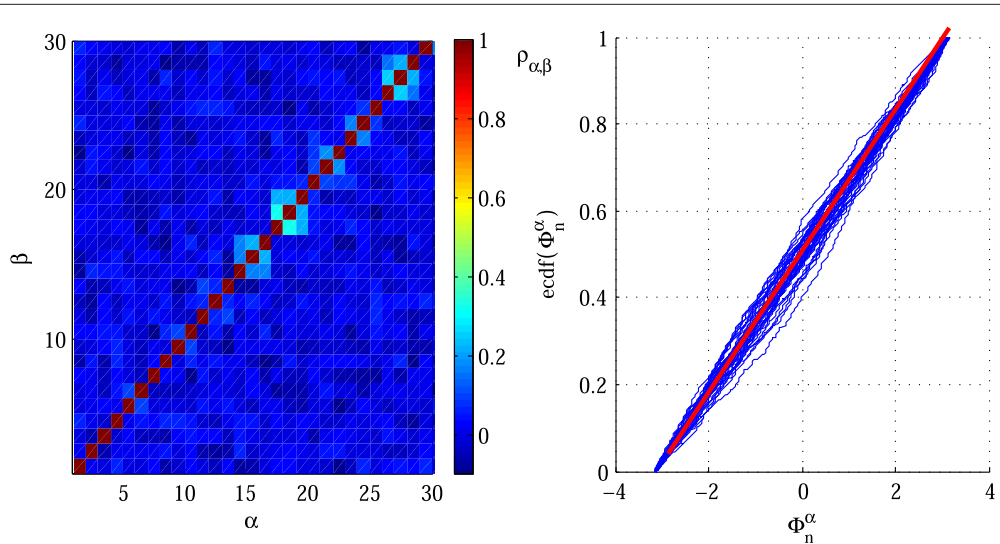

Figure 5 Pearson correlation coefficient and ecdf curves. [left] The Pearson correlation coefficient $\rho_{\alpha, \beta}$ evaluation of the measured $\Phi^{\alpha}(\tau)$. [right] The ecdf curves of $\Phi_{n}^{\alpha}$. The closer the blue ecdf curve to the red line, the closer the probability distribution of $\Phi^{\alpha}(\tau)$ to the uniform distribution. 
According to the results presented in Figure 5, we conclude that $\Phi^{\alpha}(\tau)$ is iid uniformly distributed with respect to the measurement number $\alpha$.

\subsubsection{Statistics of the received signal magnitudes and GEV}

Utilizing MLEs [21], we have found a statistical model of received signal magnitudes. As seen in Figures 6 and 7, the received signal magnitudes can be approximated using the GEV distribution [22] with the PDF given by:

$f(x \mid 0, \mu, \sigma)=\frac{1}{\sigma} \exp \{-z-\exp (-z)\}$, where $z=\frac{x-\mu}{\sigma}$,

with $\mu$ being the location parameter and $\sigma$ the distribution scale parameter. Equation 5 represents the GEV type I distribution, also known as log-Weibull distribution, where the shape parameter defined in the regular GEV is set to zero. This approach is justified in Section 3.1.3.

In order to capture the statistical nature of the environment, we have performed 90 measurements permuting both the TX and RX antenna placements as well as the incar seat occupancy. In Figure 6, we can see the CDF curves for all permutations of the antenna placement and occupancy, while each curve is fitted by a GEV type I random process obtained by the MLE fitting.

\subsubsection{GEV parameters as a random process}

According to the observations of resulting GEV parameters, we approximate the corresponding $\mu, k$, and $\sigma$ parameters with the statistical model obtained by MLE. Figures 8, 9, and 10 compare the CDFs of the measured $\mu, k$, and $\sigma$ parameters with random processes of corresponding distributions.

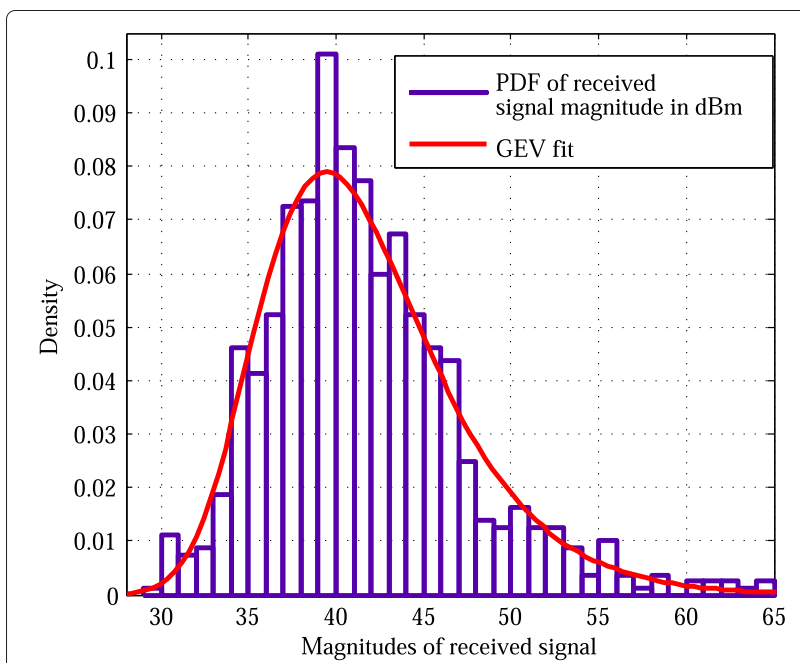

Figure 7 PDF of the received signal magnitudes in $\mathrm{dBm}$ for one measurement setup. The measured PDF is fitted with the GEV type I procedure.

The location parameter $\mu$ follows the lognormal distribution given as:

$$
f(x \mid \eta, v)=\frac{1}{x \eta \sqrt{2 \pi}} \exp \left(\frac{-(\ln x-v)^{2}}{2 \eta^{2}}\right),
$$

where $v$ is the mean and $\eta$ represents the standard deviation. The extracted shape parameter $k$ is of significantly low values; therefore, our choice of the GEV type I (also known as $\log$-Weibull) characterized by $k=0$ is appropriate (see Equation 5). The scale parameter $\sigma$ is normally distributed.

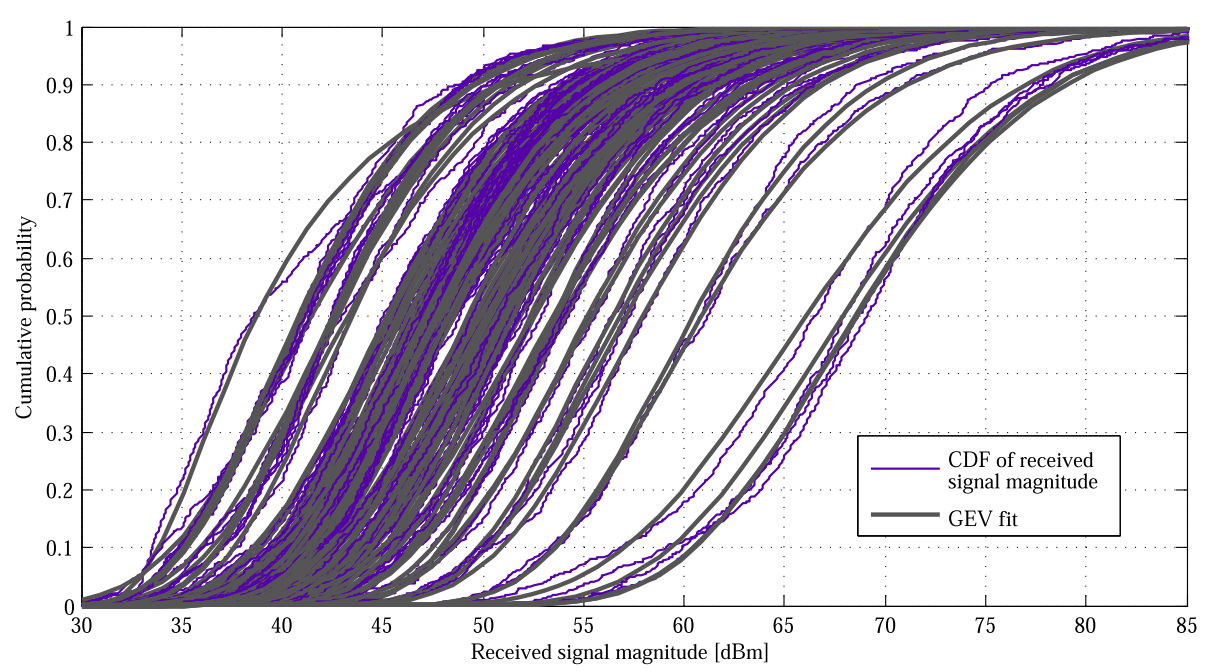

Figure 6 The CDF of received signal magnitude in dBm for all permutations of antenna placement and occupancy. Measured curves are fitted with the GEV type I procedure. 


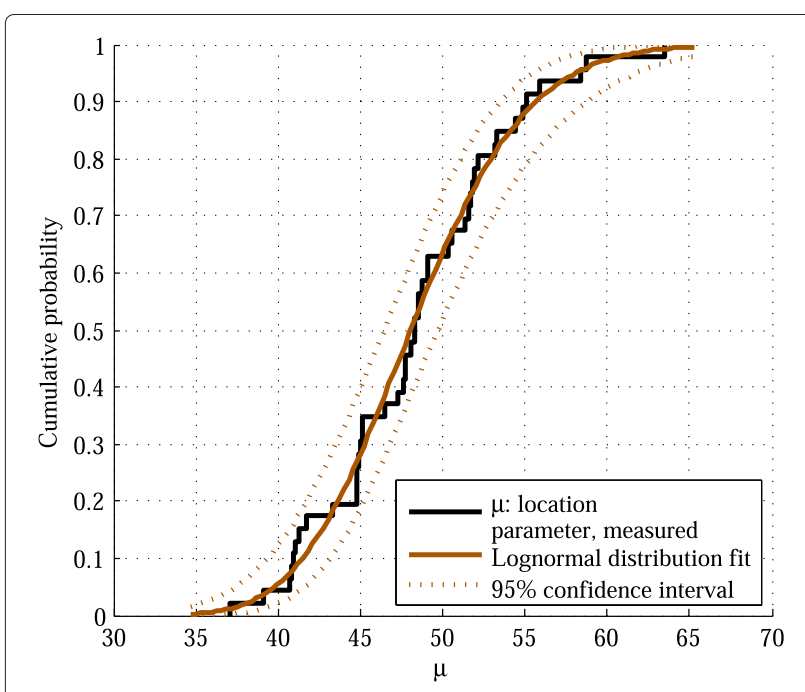

Figure 8 The CDF plot of location parameter $\boldsymbol{\mu}$ fitted with lognormal distribution (with 95\% confidence interval).

A summarized overview, including the specific values of $\mu$ and $\eta$, is given in Table 1 . Thanks to a high number of performed measurements, the tabulated values represent typical data for an in-vehicle channel which also applies to vehicles of similar size, seat configurations, and materials utilized for its manufacture.

A correlation between the derived parameters $\mu$ and $\eta$ $(k=0)$ exhibits a very weak positive correlation value of 0.35 with a $p$ value below $6 \times 10^{-4}$. Thus, to recreate the received signal magnitudes, one can arbitrarily choose the parameters $\mu$ and $\eta$ according to Table 1 .

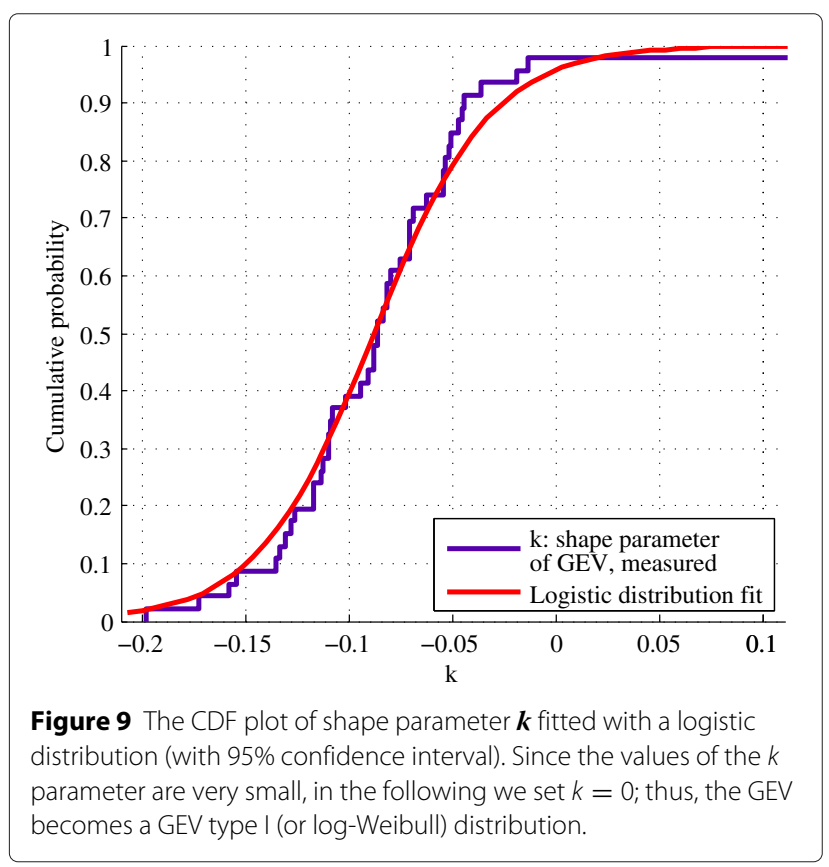

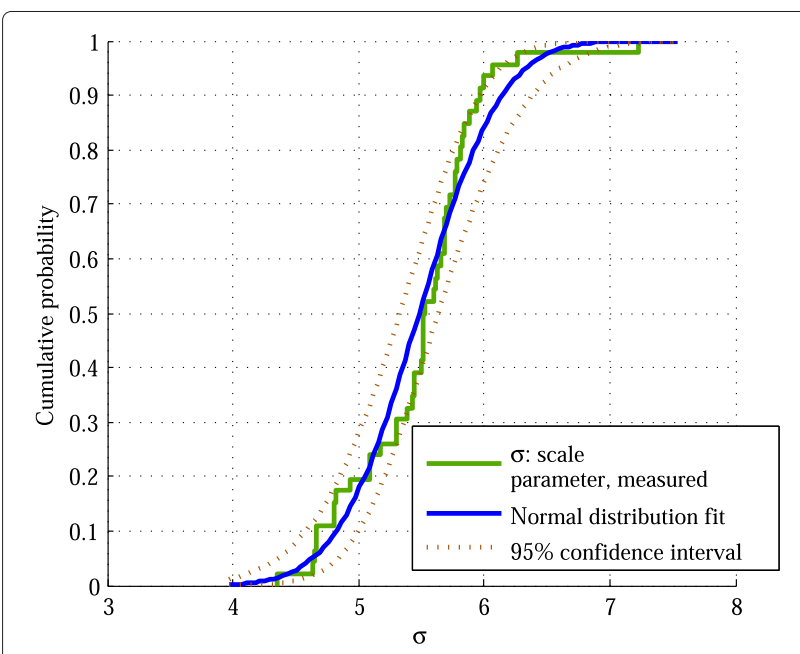

Figure 10 The CDF plot of scale parameter $\boldsymbol{\sigma}$ fitted with a normal distribution (with 95\% confidence interval).

Due to a high flexibility of the GEV fit, which is given by three input parameters as opposed to usual two parameters, the MLE metric recommends the GEV distribution. On other hand, authors in [23] claim that there is no theoretical explanation for encountering this distribution type. We may, however, add that the GEV contains the accepted $\log$-Weibull distribution as a special case for $k=0$.

\section{Localization}

One of the often discussed UWB applications is precise ranging and localization especially when the TOA technique is used. As mentioned above, this is because the large UWB bandwidth allows excellent time resolution (see Equation 7) and MPC separation. Because we had measured the channel transfer function for many different antenna positions, we wanted to get some insight into attainable ranging accuracy. Our estimation of the distance results from the CIR calculated from the complex transfer function. This approach gives some limitations compared to a direct channel sounding in the time domain where some advanced techniques such as the matched filtering of the known Gaussian pulses or a well-correlated binary sequences can be used [24].

We calculated the antenna distance using the TOA technique based on the detection of the first ray transmitted

Table 1 Summarization of GEV type I parameters characterizing in-vehicle environment for 90 permutations of antenna placement and car seat occupancy

\begin{tabular}{lccc}
\hline & $\boldsymbol{\mu}$ & $\boldsymbol{\sigma}$ & $\boldsymbol{k}$ \\
\hline Distribution type & Lognormal & Normal & Logistic \\
Mean $v$ & 48.37 & 5.48 & -0.08 \\
Variance $\eta$ & 31.05 & 0.26 & 0.002 \\
\hline
\end{tabular}


from a particular antenna. The proposed threshold-based search algorithm compares individual signal samples of the CIR with a certain threshold in order to identify the amplitude peak corresponding to the first MPC. This approach allows to calculate distance also in the NLOS scenario because the first ray may not be the strongest ray. However, penetration of the obstacles can cause some measurement accuracy degradation (see below). The aim of this chapter is to give a basic idea about accessible average error and standard deviation of the measured distances for the entire UWB band and for the first band group and also for the empty and occupied car. For more information about the measurements and the distance calculation, see [25]. Because all the particular measurements were done for three TX antennas and one RX antenna, we also calculated RX antenna position using the $2 \mathrm{D}$ localization technique in order to assess whether it corresponds at least roughly to reality, i.e., whether it is possible for example to reliably detect a device on a particular seat. Note that most of the above mentioned application does not need an accurate localization but only rough estimation of the device position.

\subsection{Basic system parameter calculation}

The frequency band $B$ determines the time resolution of measurement by [24]:

$$
T_{r}=\frac{1}{B}=\frac{1}{F_{U}-F_{L}},
$$

where $F_{U}$ is the upper frequency and $F_{L}$ is the lower frequency of the band. The propagation distance resolution is then

$$
D_{r}=T_{r} c
$$

where $c=2.998 \times 10^{8} \mathrm{~m} / \mathrm{s}$ is the speed of the light. The maximum measurable propagation distance depends on the number of measured frequency values $N_{M}$ inside the frequency band, i.e., on the frequency step $f_{s}$ according to

$$
D_{\max }=\frac{c}{B} N_{M}=\frac{c}{f_{s}} .
$$

It is obvious from the equations above that narrowing the bandwidth decreases the distance resolution and the reduction in the measured frequency points shortens the measurable propagation distance.

\subsection{Ranging and localization of the receiving antenna}

As mentioned above, the main aim of this section is calculation of the average error and standard deviation of the ranging and verification of the $\mathrm{RX}$ antenna position (Table 2). The processing of the measured data consists in the following steps:

- Calculation of the CIR

- Detection of the first incident ray

- Calculation of the RX - TX1 to TX3 distances and ranging errors

- RX antenna localization

The CIR was calculated using the IFFT in combination with a Blackman window (see Figure 11) applied to all 801 frequency response points. Before error statistics calculation, we tested a few windows (rectangular, Hann, Hamming, flattop, Blackman, and Kaiser-Bessel). Although some windows (e.g., rectangular) are generally recommended for the applications where good component separation is required, these windows could be inapplicable in our case as they may produce large side lobes that cross the threshold and cause incorrect first ray detection. Experimentally, we found that the best results giving the distances closest to reality are given by the Blackman window. The second best results can be then obtained using the Hann window.

The threshold for the first ray detection is generally determined by the noise floor. Its value is equal to the level of the peaks of noise, i.e., to the maximum amplitude of the CIR where the multipath component amplitudes are below noise level. It is obvious that the proposed algorithm works reliably in both LOS and NLOS scenarios, but it fails in some NLOS cases when the first (direct) ray is strongly attenuated and drowned in noise.

The distance of RX and TX antennas is given by the formula $D_{A}=c T_{D}$, where $T_{D}$ is the first detected ray arrival time. The error statistics were calculated separately for the empty and occupied car. It was experimentally discovered that the two or three passengers sitting in the car compartment cause very similar results, and therefore, these cases were joined into one set of results. For the RX antenna localization, the trilateration technique [24] was applied. Using the three calculated distances, this technique allows 2D localization.

Calculation of the average error and standard deviation of the measured distances is summarized in Table 3 (for

Table 2 The parameters used for the ranging

\begin{tabular}{lllllll}
\hline & $\begin{array}{l}\text { Bandwidth } \\
{[\mathrm{GHz}]}\end{array}$ & $\begin{array}{l}\text { Freq. step } \\
{[\mathrm{MHz}]}\end{array}$ & $\begin{array}{l}\text { Time resolution } \\
{[\mathrm{ns}]}\end{array}$ & $\begin{array}{l}\text { Distance } \\
\text { resolution }[\mathrm{cm}]\end{array}$ & $\begin{array}{l}\text { Max. propag. } \\
\text { distance [m] }\end{array}$ & $\begin{array}{l}\text { Max. measurement } \\
\text { time [ns] }\end{array}$ \\
\hline UWB & 8 & 10 & 0.125 & 3.750 & 30 & 100 \\
First band group & 1.58 & 0.633 & 18.987 & & \\
\hline
\end{tabular}



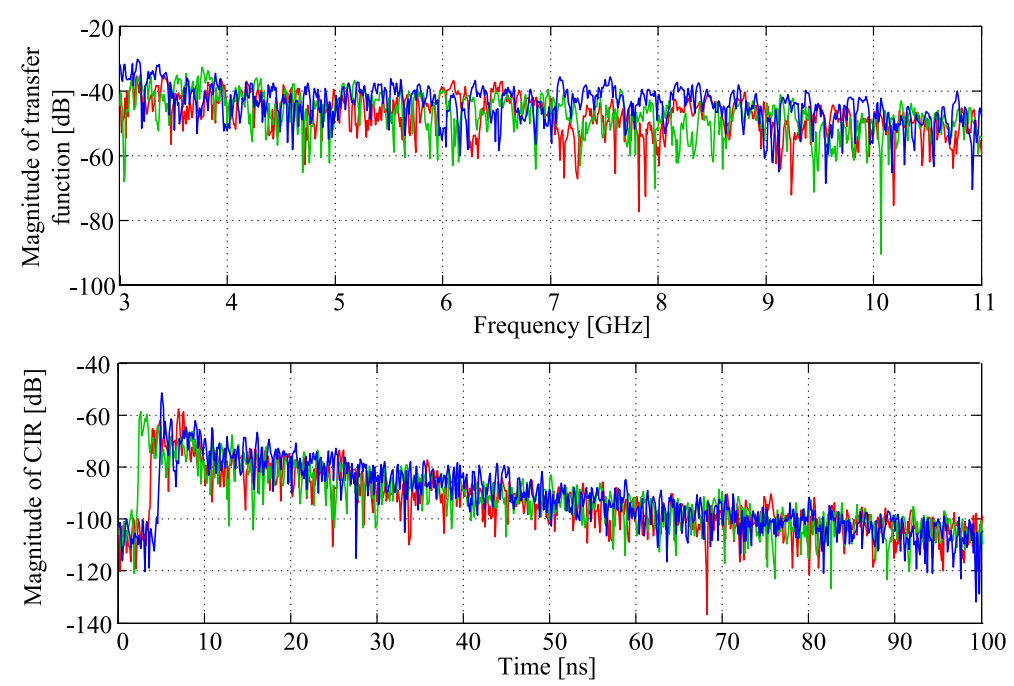

Figure 11 Magnitudes of channel transfer functions and channel impulse responses (RX antenna was placed on right rear seat).

the entire UWB) and Table 4 (for the first band group). The time intervals used for the noise peak detection were 0 to $1.25 \mathrm{~ns}$ (before receiving of the first MPC) and 80 to $100 \mathrm{~ns}$ (where the MPC can be neglected). These time intervals correspond to the following distances: 0 to 37.5 $\mathrm{cm}$ (minimum distance of RX-TX antennas in all scenarios is $50 \mathrm{~cm}$ ) and 24 to $30 \mathrm{~m}$. The reference antenna distances were measured by a ruler. We compared $15 \times 3$ distances without passengers and $15 \times 3$ distances with two or three passenger sitting on the seats surrounding the $\mathrm{RX}$ antenna. An example of peak detection for the empty car is shown in Figure 12 (upper part for the entire UWB and lower part for the first band group), while Figure 13 depicts the 2D localization result also for the UWB and first band group.

\subsection{Positioning results and sources of error}

It is obvious that the rough distance resolution in the case of the first band group measurement causes markedly higher average error and standard deviation compared to the measurement of the entire UWB band. The calculated

Table 3 Average error and standard deviation of the measured distances for the first band group

\begin{tabular}{lcccc}
\hline & TX1 & TX2 & TX3 & Total \\
\hline $\begin{array}{l}\text { Average error without passengers } \\
{[\mathrm{cm}]}\end{array}$ & 6.76 & 6.30 & 5.75 & 6.27 \\
$\begin{array}{l}\text { Average error with two or three } \\
\text { passengers [cm] }\end{array}$ & 11.83 & 10.37 & 7.62 & 9.94 \\
$\begin{array}{l}\text { Standard deviation without } \\
\text { passengers [cm] }\end{array}$ & 7.49 & 6.86 & 2.10 & 5.87 \\
$\begin{array}{l}\text { Standard deviation with two or } \\
\text { three passengers [cm] }\end{array}$ & 11.13 & 9.28 & 8.95 & 9.80 \\
\hline
\end{tabular}

distances exhibit noticeable positive bias caused by a few phenomena:

- Existence of difference between the calibration plane and phase center of the antenna. The coaxial interfaces of the antennas (line between the connector and phase center of the antenna) were not included when the VNA was calibrated. They were applied only during channel measurement and increased the total antenna distance.

- Inaccurate reference measurement. Distance measured between the antennas by the ruler was performed between the centers of the top of cones which are not identical to the phase centers of antennas. In many cases, the measured distance were slightly shorter (when the TX antenna was upside down with regard to RX antenna) .

- Time lag in the first ray detection. The first ray (peak) detection above the threshold exhibits random delay in the interval 0 to $D_{r}$ due to the discrete nature of the CIR time axis. Received ray cannot be generally detected in advance.

Table 4 Average error and standard deviation of the measured distances for the first band group

\begin{tabular}{lcccc}
\hline & TX1 & TX2 & TX3 & Total \\
\hline $\begin{array}{l}\text { Average error without passengers } \\
{[\mathrm{cm}]}\end{array}$ & 25.85 & 21.04 & 14.3 & 20.39 \\
$\begin{array}{l}\text { Average error with two or three } \\
\text { passengers [cm] }\end{array}$ & 34.73 & 23.50 & 10.82 & 23.02 \\
$\begin{array}{l}\text { Standard deviation without } \\
\text { passengers [cm] }\end{array}$ & 20.76 & 13.88 & 8.74 & 15.67 \\
$\begin{array}{l}\text { Standard deviation with two or } \\
\text { three passengers [cm] }\end{array}$ & 20.13 & 12.63 & 7.88 & 17.26 \\
\hline
\end{tabular}



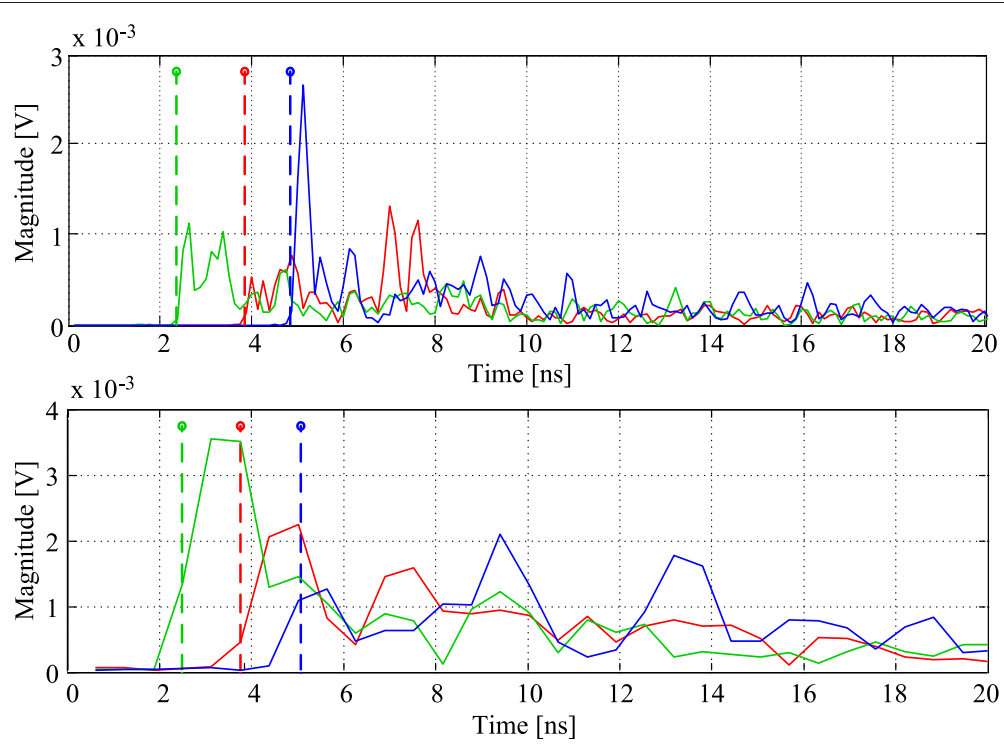

Figure 12 First peak detection of CIRs (for entire UWB) [upper] for empty and [lower] occupied vehicle.

- Incorrect MPC component detection. Large attenuation of some obstacles in the car may avoid correct detection of the direct ray. In this case, the other reflected MPC which travels on a longer path is regarded as the first ray.

- Lower wave propagation velocity in media. The velocity of an electromagnetic wave penetrating an obstacle is less than that in free space, and it depends on the obstacle material constants.

The first phenomenon is systematic and can be subtracted (it is about $2 \mathrm{~cm}$ together for two antennas). The two last phenomena occur only in the NLOS scenario. In the last case, the velocity in some material can be calculated according the formula $v_{p}=c / \sqrt{\varepsilon_{r} \mu_{r}}$, where $v_{p}$ is the velocity of propagation in $\mathrm{m} / \mathrm{s}, \mu_{r}$ is the material relative permeability, and $\varepsilon_{r}$ is the relative permittivity. It is easy to find that when, for example, the wave passes the 10-cm-thick plastic obstacle $\left(\varepsilon_{r}=2\right.$ to $3, \mu_{r}=1$ [26]), the propagation time delays are in the interval 0.138 to 0.244 ns which results in the distance bias from 4.1 to $7.3 \mathrm{~cm}$.

\section{Conclusions}

We performed an extensive UWB measurement campaign for the vehicular passenger compartment. The measured

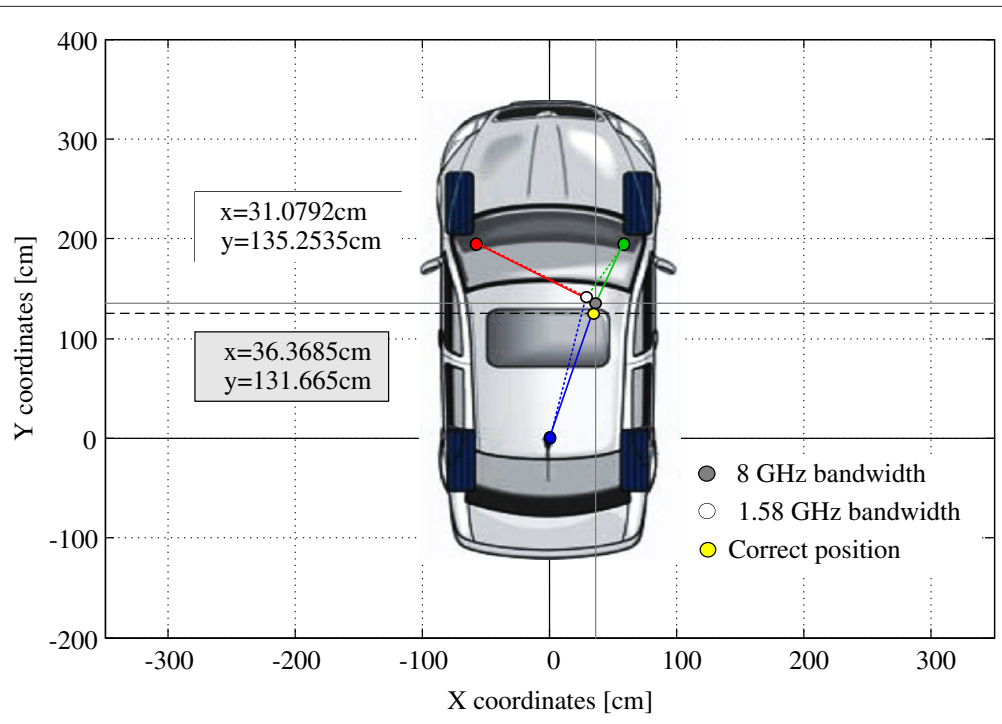

Figure 13 Localization of the RX antenna using TOA (RX antenna was placed on front passenger seat). 
channel impulse responses are modeled using the GEV distribution; its parameters are estimated using a MLE. As a result, our statistical description of the received amplitude and phase distribution in the in-vehicle environment fits almost perfectly to the empirical measurement results. We showed that the measured phase is uniformly distributed with iid behavior.

Based on the measurement data, a feasibility study on the use of UWB-based positioning inside the vehicle was conducted. We could show that the accuracy of the transmitter location could be obtained with a standard deviation smaller than $10 \mathrm{~cm}$ for the full UWB bandwidth. The standard deviation was smaller than $16 \mathrm{~cm}$ for the first UWB band group only. The influence of the antenna position on the localization accuracy was lower than the effect of the occupancy level of the car.

\section{Competing interests}

The authors declare that they have no competing interests.

\section{Acknowledgements}

This work was supported by the Czech Science Foundation Project No. 13-38735S Research into wireless channels for intra-vehicle communication and positioning. Research described in this paper was financed by Czech Ministry of Education in frame of National Sustainability Program under grant LO1401. For research, infrastructure of the SIX Center No. CZ.1.05/2.1.00/03.0072 was used. The cooperation in the COST IC1004 action was supported by the MEYS of the Czech Republic Project No. LD12006 (CEEC).

\section{Author details}

${ }^{1}$ Department of Radio Electronics, Brno University of Technology, Technicka 12,612 00, Brno, Czech Republic. ${ }^{2}$ AlT Austrian Institute of Technology GmbH, Donau-City-Straße 1, 1220 Vienna, Austria. Institute of Telecommunications, Vienna University of Technology, Gußhausstraße 25/E 389, 1040 Vienna, Austria.

Received: 15 September 2014 Accepted: 5 March 2015

Published online: 15 April 2015

\section{References}

1. G Leen, D Heffernan, Expanding automotive electronic systems. Computer. 35(1), 88-93 (2002)

2. M Win, R Scholtz, Characterization of ultra-wide bandwidth wireless indoor channels: a communication-theoretic view. Selected Areas Commun. IEEE J. 20(9), 1613-1627 (2002)

3. R-M Cramer, R Scholtz, M Win, Evaluation of an ultra-wide-band propagation channel. Antennas Propag. IEEE Trans. 50(5), 561-570 (2002)

4. M Schack, J Jemai, R Piesiewicz, R Geise, I Schmidt, T Kurner, in IEEE Vehicular Technology Conference, 2008. Measurements and analysis of an in-car UWB channel (VTC Spring 2008 Singapore, 11-14 May 2008), pp. 459-463

5. T Kobayashi, in 2006 IEEE Ninth International Symposium on Spread Spectrum Techniques and Applications, Manaus-Amazon. Measurements and characterization of ultra wideband propagation channels in a passenger-car compartment, (28-31 Aug 2006), pp. 228-232

6. T Tsuboi, J Yamada, N Yamauchi, in 7th International Conference on ITS Telecommunications, 2007. ITST'07. UWB radio propagation inside vehicle environments (Sophia Antipolis, 6-8 June 2007), pp. 1-5

7. M Schack, R Geise, I Schmidt, R Piesiewiczk, T Kurner, in 3rd European Conference on Antennas and Propagation, 2009. EUCAP 2009. UWB channel measurements inside different car types (Berlin, 23-27 March 2009), pp. 640-644

8. A Moghimi, H-M Tsai, C Saraydar, O Tonguz, Characterizing intra-car wireless channels. Vehic. Technol. IEEE Trans. 58(9), 5299-5305 (2009)
9. J Blumenstein, T Mikulasek, R Marsalek, A Prokes, T Zemen, C Mecklenbrauker, in 2014 IEEE 80th Vehicular Technology Conference (VTC Fall). In-vehicle mm-wave channel model and measurement (Vancouver, BC, 14-17 Sept 2014), pp. 1-5

10. PC Richardson, W Xiang, W Stark, Modeling of ultra-wideband channels within vehicles. Selected Areas Commun. IEEE J. 24(4), 906-912 (2006)

11. L Liu, Y Wang, N Zhang, Y Zhang, in 2010 12th IEEE International Conference on Communication Technology (ICCT). UWB channel measurement and modeling for the intra-vehicle environments (Nanjing, 11-14 Nov 2010), pp. 381-384

12. B Li, Z Zhou, D Li, S Zhai, Efficient cluster identification for measured ultra-wideband channel impulse response in vehicle cabin. Prog. Electromagnetics Res. 117, 121-147 (2011)

13. J Li, T Talty, in Military Communications Conference, 2006. MILCOM 2006. Channel characterization for ultra-wideband intra-vehicle sensor networks (Washington, DC, 23-25 Oct 2006), pp. 1-5

14. R Thoma, O Hirsch, J Sachs, R Zetik, in The Second European Conference on Antennas and Propagation, 2007. EUCAP 2007. UWB sensor networks for position location and imaging of objects and environments (Edinburgh, 11-16 Nov 2007), pp. 1-9

15. J Blumenstein, R Marsalek, A Prokes, C Mecklenbrauker, in Multiple Access Communcations. Impulse noise mitigation for OFDM by time-frequency spreading, vol. 8310 (ser. Lecture Notes in Computer Science. Springer International Publishing Vilnius, Lithuania, 16-17 Dec 2013), pp. 8-20

16. J Blumenstein, T Mikulasek, R Marsalek, A Chandra, A Prokes, T Zemen, C Mecklenbrauker, in IEEE Vehicular Networking Conference (VNC). In-vehicle UWB channel measurement, model and spatial stationarity (Paderborn, 3-5 Dec 2014), pp. 77-80

17. R Fontana, E Richley, J Barney, in 2003 IEEE Conference on Ultra Wideband Systems and Technologies. Commercialization of an ultra wideband precision asset location system (Reston, VA, USA, 16-19 Nov 2003), pp. 369-373

18. R-R Lao, J-H Tarng, C Hsiao, in The 57th IEEE Semiannual Vehicular Technology Conference, 2003. VTC 2003-Spring. Transmission coefficients measurement of building materials for UWB systems in 3-10 GHz, vol. 1 (Jeju, Korea, 22-25 April 2003), pp. 11-14

19. Q Liang, A Audu, H Khani, H Nie, W Xiang, Z Chen, in 2013 IEEE Radio and Wireless Symposium (RWS). Measurement and analysis of intra-vehicle UWB channels (Austin, TX, USA, 20-23 Jan 2013), pp. 166-168

20. JD Kraus, McGraw-Hill Education, (New York, 1988)

21. RA Fisher, in Mathematical Proceedings of the Cambridge Philosophical Society. Theory of Statistical Estimation, (1925), pp. 700-725

22. S Kotz, S Nadarajah, Extreme Value Distributions, (World Scientific, Washington, D.C, 2000)

23. H Hashemi, The indoor radio propagation channel. Vehicular Technology, IEEE Transactions on. 81(7), 594-606 (2002)

24. Z Sahinoglu, S Gezici, I Guvenc, vol. 2. (Cambridge University Press, Cambridge, 2008)

25. J Vychodil, J Blumenstein, T Mikulasek, A Prokes, V Derbek, in International Conference on Connected Vehicles \& Expo 2014 ICCVE 3rd. Measurement of in-vehicle channel feasibility of ranging in UWB and MMW band (Vienna, 3-7 Nov 2014)

26. DR Lide, CRC Handbook of Chemistry and Physics. (CRC Press, 2001) 